\section{Severe Growth Failure and Poorly Controlled Type 1 Diabetes Mellitus in a 7-Year-Old Girl: Mauriac Syndrome}

Sir,

Insulin dependent type 1 diabetes mellitus (T1DM) is an important cause of admission in pediatric ward. Newly diagnosed diabetics usually fall in the age group from 2 years to 7 years. Well known complications of diabetes are ketoacidosis, hypoglycemia, and infections. Although growth may be compromised in poorly controlled T1DM, ${ }^{1}$ severe stunting of growth associated with poor compliance to insulin regimen and caloric restriction is a rare finding manifested in these children as Mauriac syndrome. Conditions predisposing T1DM children to this complication are short-acting insulin regimens, poor compliance, and severe caloric restrictions. Associated findings are hepatomegaly and elevated serum transaminases along with cushingoid facies, which is an unusual feature of grade three undernutrition. ${ }^{2}$ Mauriac syndrome was described for the first time in 1947.3 Although still witnessed in developing countries, it is a rare finding in developed countries due to use of longacting preparations of insulin, a variety of insulin administration techniques, better storage facilities, and better glycemic control.

We report here a case of Mauriac syndrome, a rare complication associated with T1DM. A 7-year girl with known T1DM since five years, (she was diagnosed at two years of age), was admitted in Pediatrics Ward at Ayub Teaching Hospital. She had high grade fever secondary to respiratory tract infection for four days. Her random blood sugar at admission was $402 \mathrm{mg} / \mathrm{dl}$. She was not on a proper regimen of insulin as she was injected with insulin only once daily. There was improper storage of insulin; and injection sites, mostly on abdomen, appeared to be infected. Due to multiple problems, she was non-compliant with the treatment, and was previously once admitted with ketoacidosis. The child was being given a combination of intermediate and short-acting insulin in 70:30 proportions in only once daily dose. Glycemic control was poor as evident by $\mathrm{HbA} 1 \mathrm{c}$ levels which were $13.2 \%$ performed at admission. Mother was concerned about her growth and extremely poor appetite. Her growth percentiles were arrested at same measurements as they were at two years of age, and calorie intake was very restricted. All growth

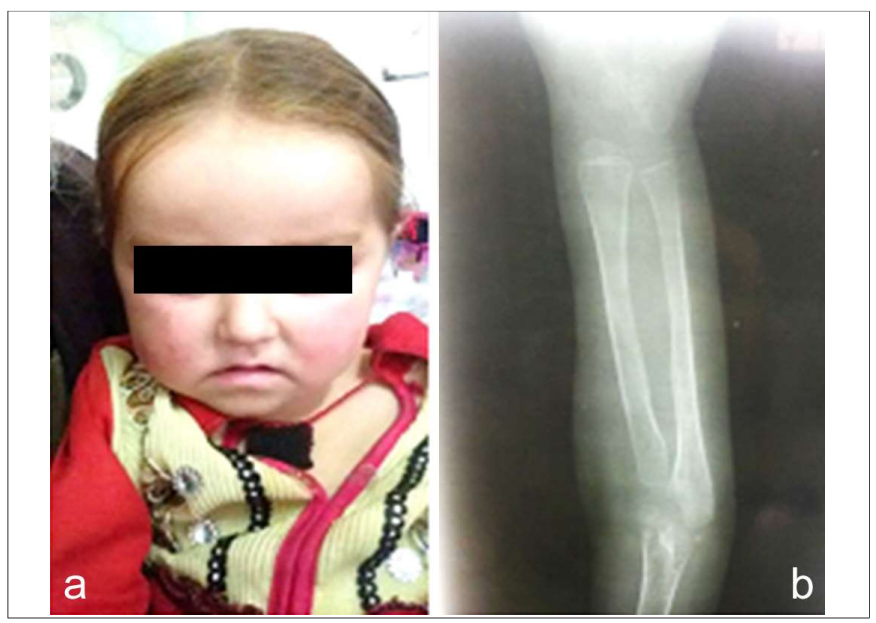

Figure 1: (a) Face of a patient showing Cushing's like features. (b) X-ray of left arm wrist joint and delay in bone age as only 3 carpal bones are visible at seven years of age.

percentiles fell below the $5^{\text {th }}$ percentile. At seven years, her mid-upper arm circumference was $11 \mathrm{~cm}$, height was $78 \mathrm{~cm}$, weight was $10.1 \mathrm{~kg}$, and head circumference was $46 \mathrm{~cm}$. Facial features included a moon face, and malar erythema which are characteristic of Cushing's like facies and not an expected feature in T1DM (Figure 1a). Insulin injection sites, mostly found on the abdomen around the umbilicus and the thighs, were infected and exhibited features of lipodystrophy and lipohypertrophy.

Abdomen was protuberant. There was hepatomegaly, and edge of liver was palpable $3 \mathrm{~cm}$ below costal margin. Arms and legs showed proximal muscle wasting and atrophy. Due to stunted growth and the association of T1DM with other autoimmune disorders, thyroid function tests were done, but serum levels of TSH and T4 showed in normal ranges. Complete blood picture showed only leukocytosis count, probably due to recent acute infection. Ultrasound scan of the abdomen showed enlarged liver with a normal echo texture. Serum ALT levels were raised. Radiological findings included a delayed bone age (Figure 1b). As the patient was from a mountainous village so there was no electricity to store insulin preparation. Patient had no knowhow about storage of insulin. Dosage and dietary compliance was unsatisfactory that led her to severe malnourishment, growth stunting, and Mauriac syndrome. Initially, we started on a 6-hourly dosage of 2 units of regular insulin; diet was reviewed, and calories built up.

Re-counselling was done regarding the insulin administration and patient was sent home after shifting to a 70:30 BID insulin regimen.

Growth stunting in T1DM can be due to a number of causes. Poor glycemic control disrupts the insulingrowth hormone axis; and strict dietary restriction and non-compliance further complicates the situation. 
Association with childhood illnesses such as cystic fibrosis, celiac disease and hypothyroidism as part of autoimmune polyglandular syndrome can also led to poor growth; plus failure to thrive and need to be ruled out as possible causes of growth failure. 4 Liver biopsy in Mauriac, syndrome can demonstrate steatosis as well as glycogen deposition and altered level of transaminases.

As all children with type 1 diabetes do not develop Mauriac, so a genetic possibility is linked to it. ${ }^{5}$ Compliance among type 1 diabetics and ensuring optimal growth and sufficient caloric intake is a problem as with most pediatric chronic illnesses. Dealing with, and trying to explain the disease, its management and outcome to uneducated parents makes the situation even more difficult. Achieving optimal glycemic control in T1DM faces many challenges in Pakistan such as restricted choices in various insulin delivering devices, expensive long acting insulin preparations, poverty, difficult access to hospitals and professional health personnel, no road access to mountainous Northern areas, absence of electricity, and power outages; hence, making storage of insulin difficult. Complicated disease, like T1DM, requires meticulous counselling and strict follow-ups that can be made possible, if pediatric endocrinologist, dieticians and general pediatric specialists work together. Availability of economical insulin preparations, proper storage, dietary compliance, home monitoring of glucose levels, and accessibility to health personnel are challenges that need to be tackled with.

\section{REFERENCES}

1. Giannini C, Angelika M, Chiarelli F. Growth abnormalities in children with type 1 diabetes, juvenile chronic arthritis, and asthma. Int $J$ Endocrinol 2014; 265954.

2. Palacios CM, Vera JP, Chinchilla JF, Marco JF, Galindo MA, Ferrer LG. Hypertransaminasemia in poorly-controlled type-1 diabetes mellitus. Rev Esp Enferm Dig 2004; 96:730-1.

3. Patidar P, Philip R, Saran S, Gupta KK. A rare case of Mauriac syndrome. Indian J Endocrinol Metab 2012; 16:486-7.

4. Hunger-Battefeld W, Fath K, Mandecka A, Kiehntopf M, Kloos C, Müller UA, et al. Prevalence of polyglandular autoimmune syndrome in patients with diabetes mellitus type 1. Med Klin (Munich) 2009; 104:183-91.

5. MacDonald MJ, Hasan NM, Ansari IU, Longacre MJ, Kendrick MA, Stoker SW. Discovery of a genetic metabolic cause for mauriac syndrome in type 1 diabetes. Diabetes 2016; 65:2051-9.

Saima Gillani ${ }^{1}$, Attia Iqball and Nasir Hussain Shah Kazmi ${ }^{2}$

Department of Pediatrics ${ }^{1}$ / Medicine ${ }^{2}$, Ayub Medical College, Abbottabad, Pakistan

Correspondence: Dr. Saima Gillani, Department of Pediatrics, Ayub Medical College, Abbottabad, Pakistan E-mail:drsaimagillani@gmail.com

Received: August 07, 2018; Accepted: December 4, 2018 